\title{
Diabetes Neonatal Transitoria
}

\author{
Dra Gladys Bórquez E. ${ }^{1}$; Dra. Alejandra Reyes J. ${ }^{2}$ \\ Transient Neonatal Diabetes
}

\begin{abstract}
Transient neonatal diabetes was noted in a small for date newborn baby, at the age of 42 days. The clinical manifestations tipical of this disease were present in this girl, and through them it was possible to make the diagnosis. She did not required theraphy with insulin, recovering spontaneously. She was controlled after 9 months and found healthy. An analysis of the differential diagnosis is done and also a comentary on the treatment, aetiology, pathogenesis and heredity based on the revision of the publications about the subject.
\end{abstract}

La Diabetes Neonatal Transitoria es un trastorno caracterizado por hiperglicemia y glucosuria, que afecta predominantemente a los recién nacidos pequeños para la edad gestacional, se presenta durante las primeras seis semanas de vida, poco frecuente, su incidencia, se desconoce, su origen y herencia tampoco están bien defini$\operatorname{dos}^{1}$.

Las manifestaciones clínicas más importantes son: pérdida de peso marcada; deshidratación

\footnotetext{
1 Médico Servicio de Pediatría Hospital Félix Bulnes.
}

2 Becado Servicio de Pediatr ía Hospital Félix Bulnes. severa, a pesar de una ingesta oral adecuada; fiebre, poliuria y conservación del estado alerta sin llegar al coma ${ }^{2}$.

Entre los signos de laboratorio destacan hiperglicemia, que va desde 240 hasta $2300 \mathrm{mg} \%$ y glucosuria intensa. Habitualmente no se produce cetosis, pero puede haber acidosis metabólica, leve. La natremia puede ser normal o baja. Los valores de insulina plasmáticos son bajos cuando se miden durante la fase aguda ${ }^{\mathbf{1 - 3}}$.

La duración del trastorno es variable, desde días a meses, con un promedio de 24 meses. Su pronóstico es bueno, pues es transitorio. Se han descrito pacientes seguidos hasta 25 años sin 
nuevos problemas ${ }^{4-5-6} \mathrm{Sin}$ embargo un caso evolucionó hacia diabetes permanente?

\section{Caso Clínico}

M.C.C., de sexo femenino, producto del primer embarazo; nacida por cesárea, por retardo de crecimiento intra intrauterino y sufrimiento fetal. Edad gestacional de 38 semanas; pequeño para la edad gestacional; apgar 8 al minuto; muy enflaquecido, piel laxa y descamada, ausencia de panículo adiposo; peso $1.430 \mathrm{grs}$, talla $41 \mathrm{cms}$. y C.C. $39 \mathrm{cms}$. Madre sin antecedentes de diabetes. Se ignoran antecedentes paternos.

Ingresó a los 30 minutos de vida a la Unidad de Neonatología del Hospital Félix Bulnes con hjpoglicemia de $30 \mathrm{mg} \%$ que se trató con glucosa endovenosa, normalizándose rápidamente. $\mathrm{Re}$ cuperó su peso de nacimiento al décimo día de vida y se fue de alta a los 38 días, con $2.240 \mathrm{~g}$., de peso, subiendo 30 a 40 g. por día sin proble. mas.

Reingresó a Neonatología 5 días después, a los 42 de vida, por decaimiento y rechazo parcial de la alimentación desde 48 horas antes, con deshidratación intensa, pérdida de $15 \%$ de su peso (1.900 grs., peso jngreso), fiebre moderada, respiración profunda, llanto débil, sin vómitos ni diarrea, orina diluida y poliuria. Glucosuria intensa, glicemia preprandial de 390 y $618 \mathrm{mg} \mathrm{x}$ dl; $\mathrm{pH}$ sanguíneo $7.336 \mathrm{pCO}_{2} 21 \mathrm{~mm} \mathrm{Hg}$, bicarbonato $10 \mathrm{~m} . \mathrm{mol} \times 1$. Exceso de Base (E.B.) $-11,3$ $\mathrm{mEq} \times 1$, sin cuerpos cetónicos en sangre ni orina. Pruebas de función renal normales.

Se trató con hidatación parenteral usando soluciones glucosalinas hipotónicas, con cargas de glucosa endovenosa bajas (hasta $1,3 \mathrm{mg} / \mathrm{kg} / \mathrm{min}$.). Evolucionó bien, desapareciendo la fiebre y el letargo en 24 hrs., pero siguió deshidratada por 4 días, con poliuria, glucosuria e hiperglicemia. No tuvo cetosis ni reapareció la acidosis después de las correcciones hechas al ingreso.

Al quinto día de hospitalización estaba bien hidratada, su diuresis era normal, no tenía glucosuria y se había normalizado la glicemia. Desde entonces se alimentó por vía oral con fórmula láctea en volumen creciente, hasta alcanzar 173 cal. $/ \mathrm{kg} /$ día con lo que se obtuvo aumentos diarios de peso de 47 grs. e incremento de $3,5 \mathrm{~cm}$., de la talla en un mes.

Dada de alta a los 64 días de edad se ha mantenido asintomática, con glicemias pre y postprandiales normales (72 a $96 \mathrm{mg} \%$ ) y sin glucosuria, con cuatro meses de seguimiento.

\section{COMENTARIOS}

Las características clínicas de nuestro caso concuerdan con las comunicadas desde la primera publicación sobre Diabetes Neonatal Transitoria en 1929. El diagnóstico diferencial se desarrolla a partir de la existencia de glicemia elevada ${ }^{3}$.

La hiperglicemia puede, en algunos casos, ser secundaria al uso de cargas inadecuadas de glu. cosa endovenosa, pues se sabe que la tolerancia por la glucosa está alterada en los prematuros y desnutridos intrauterinos, según se postula, por persistente producción de glucosa hepática endógena ${ }^{a}$. Con el uso ampliamente difundido de la nutrición parenteral total, se ha podido conocec en los recién nacidos de bajo peso su tolerancia a la infusión endovenosa; así se dice que los recién nacidos de término, como los lactantes, toleran inicialmente cargas de 7 a $8 \mathrm{mg} \mathrm{x} \mathrm{kg} \mathrm{y} \mathrm{min,}$ pudiendo rápidamente aumentar hasta 12 a 14 $\mathrm{mg} \times \mathrm{kg} \times \mathrm{min}$. sin dificultad. Para los prematuros menores de $1.000 \mathrm{grs}$, se aconseja no dar más de $6 \mathrm{mg} x \mathrm{~kg} \mathrm{x}$ min y entre 1.000 y 1.500 grs. de peso, no pasar de $8 \mathrm{mg} \times \mathrm{kg} \times \mathrm{min}^{9}$

Otras causas frecuentes de glicemia elevada son las descritas en RN críticamente enfermos, con asfixia grave, hemorragia intracraneana o sepsis, $y$ en edades mayores en grandes quema. duras. ${ }^{10}$

También se describe la asociación de hiperglicemia con delección $46 \times X \mathrm{Xq}$ - del cromosoma 13, que se acompaña de holoprosencefalia, microsftalmia, coloboma ocular, hipoplasia de pulgares y anomalía génito-anal. ${ }^{1} 1$

No estă aún delineado con precisión el manejo de estos casos. La respuesta a la administración de insulina tiene gran variabilidad individual en el recién nacido. Algunos casos de Diabetes Neona. tal Transitoria necesitan insulina $y$ requieren dosis individuales bajas e intermitentes; en otros su empleo es innecesario, siendo sufjiciente la hidratación con soluciones hipotónicas para corregir la concentración sanguínea de glucosa ${ }^{1}$

Diversos trabajos muestran que durante la fase aguda de la enfermedad no hay liberación de insulina por las células beta pancreáticas en respuesta a la estimulación con glucosa y tolbutamida, pero sí por efecto de otros secretagogos como cafeina y teofilina, lo que ha sido interpretado como evidencia de insulina en el páncreas y de un posible defecto madurativo del sistema AMP cíclico en ese órgano 12-13-14.

Algunos autores han usado una sulfonilurea de acción prolongada como tratamiento pensando que su acción estimulante de la secreción perni. tiría acortar la duración del tratamiento y por lo tanto limitar el uso de insulina ${ }^{15}$. Conociendo el efecto de la insulina en el crecimiento, dado que los casos de Diabetes Neonatal Transitoria se ven principalmente en desnutridos intrauterinos, se ha postulado que la falta de insulina "in útero" 
sea la causa del pobre crecimiento ${ }^{16}$, al contrario de lo que ocurre con el hijo de madre diabética; corroborando esto vemos una rápida aceleración del incremento de peso y talla al mejorar la función pancreática ${ }^{7}{ }^{7}$. Aún más, algunos autores distinguen, sobre la base del peso de nacimiento, dos tipos de Diabetes Neonatal Transitoria, una genética y otra iatrogénica ${ }^{18}$.

No existe hasta ahora un patrón de herencia definido ni se conoce la relación entre Diabetes Neonatal Transitoria y Diabetes Mellitus en la infancia o edad adulta. Se ha comunicado el caso de tres hermanos de sexo masculino con Diabetes Neonatal Transitoria ${ }^{4}$. En otra serie familiar, constituída por un varón y dos mujeres hijos de un mismo padre, todos con Diabetes Neorfatal Transitoria, una de las niñas desarrolló a la edad de 15 años Diabetes insulino dependiente ${ }^{5 \cdot 6-7}$.

\section{RESUMEN}

En una niña pequeña para la edad gestacional se diagnosticó Diabetes Neonatal Transitoria a los 42 días de vida por presentar las manifestaciones clínicas características de la enfermedad.

No fue necesario tratarla con insulina, recuperándose espontáneamente. Controlada a los 9 meses y se encontró sana.

\section{REFERENCIAS}

1 Combiath, Schwartz.: Disorders of carbohydrate metabolism in infancy Second Edition. W.B. Saunders Company USA. pág. $218,1976$.

2 Hutchison J.H., Keay' A.: Congenital temporary diabetes mellitus. Br. Med. J. 2: 436, 1962.
${ }^{3}$ Gordon B.: Avery. Carbohydrate homeostasis in the fetus and newbom. In: Neonatology, Philadelphia Second Edition, pág. 592, 1981.

4 Ferguson A.. Milner R.: Transient neonatal diabetes mellitus in three successive male siblings. Arch. Dis, Child 46: 724, 1971.

s Coffey J., Womack $N$.: Transient neonatal diabetes mellitus in half sisters. Am. J. Dis. Child. 113: 480, 1967.

- Ferguson A., Milner R.: Transient neonatal diabetes mellitus Arc. Dis. 45: 80, 1970.

7 Coffey J., Killelea D.: Transient neonatal diabetes mellitus in half sisters. Am. J. Dis. Child. 136: $626,1982$.

- Cowet R., Schwortz R.: Neonatal hypo-and-hyperglycemia, Seminars in perinatology. 3: 327, 1979.

* Kerner, J., Sunshine P.: Parenteral nutrition. Seminars in perinatology. 3: 417, 1979.

10 Rosenbloom A., Hunt S.: Promosis of impaired glucose tolerance in children with stress hyperglycemia. J. Pediats. 101: $340,1982$.

11 Leisto $J$, Raivio $K$, Krohn $K$.: Neonatal hypergly. cemia and chromosomes deletion $46 \times X^{2} q-$. J. Pediatr. 88: 989, 1976.

12 Ferguson A.: Neonatal hyperglycemia, case report with plasma insulin studies. Arch. Dis. Child. 42: $509,1967$.

13 Pagliaria A., Kail L., Kipnis D.: Transient neonatal diabetes J. Pediatr. B2: $97,1973$.

14 Sodoyez-Goddaux F., Sodoyez J.: Transient diabetes mellitus in a neonate J. Pediatt. 91: 395 , 1977.

15 Kuna P., Addy D.: Transient neonatal diabetes Treatment with chlorpropamide. Am. J. Dis. Child. $133: 65,1979$.

16 Gerrard J., Chin $W_{\text {.: }}$ The syndrome of transient diabetes. J. Pediatr. 61: 89, 1962.

17 Blethen S. White: Plasma somatomedins, endogenous insulin secretion and growth in transient neonatal diabetes mellitus. J. Clin. Endocrinol. Metab. 52: 144,1981

18 MacDonal M.: Diabetes Neonatal. Lancet 1: 737 , 1974 